\title{
Reply to Dhillon et al.: Comments on Kailu et al.: Chronic perilunate dislocations treated with open reduction and internal fixation: results of medium term follow-up
}

\author{
Kailu Liang • Xiaoling Gan • Zhongliang Deng • \\ Zhou Xiang $\cdot$ Fuguo Huang
}

Received: 31 January 2011 / Accepted: 1 February 2011 /Published online: 19 February 2011

(C) Springer-Verlag 2011

Sir,

We deeply appreciated the comments from Joshi et al. concerning our article [1].

Indeed, the Geissler classification system was initially developed to differentiate various grades of ligament disruptions in wrist arthroscopic surgery [2]. However, this system provides a relatively complete description on luno-triquetral ligament and scapholunate ligament damage. Therefore, in this study, we used this system to measure the severity of these two types of ligament damage in the situation of perilunate dislocation. In most cases of trans-scaphoid perilunate dislocation, the luno-triquetral ligament tears completely to allow the triquetrum to dislocate dorsally along with the rest of the carpus (Mayfield stage III) [3]. However, in our practice we found that this ligament could be just stretched or partially torn in trans-scaphoid perilunate dislocation. In scaphoid fracture, the scapholunate ligament could also be partially damaged. Therefore, it is quite useful to apply the Geissler classification system to describe the severity of ligament damage more precisely.

\section{K. Liang $(\varangle) \cdot$ Z. Deng}

Department of Orthopaedics, The Second Affiliated

Hospital of Chongqing Medical University,

Chongqing, China

e-mail: liang-kai-lu@163.com

\section{Gan}

Department of Gynaecology, The First Affiliated

Hospital of Chongqing Medical University,

Chongqing, China

\section{Z. Xiang $\cdot$ F. Huang}

Department of Orthopaedics, Huaxi Hospital, Sichuan University, Chengdu, China
We agree that in delayed and chronic perilunate dislocation, significant fibrosis and scarring makes ligament identification difficult. Moreover, it is practically impossible to repair the ligament by direct suture. Therefore, in this study, ligamentous repair was performed with transosseous suture techniques using nonabsorbable material.

We also agree that K-wires can stabilise the lunotriquetral, capito-lunate and scapho-lunate joints after reduction. However, K-wire fixation can not provide adequate stability for early wrist functional rehabilitation (in this study, range of motion exercises were started on the third postoperative day). Thus, the wrist external fixator was used to supply more stability to realise early functional rehabilitation of the wrist. Moreover, the application of the wrist external fixator in a neutralising mode can help to protect the cartilage of the carpal bones by blocking the axial load during functional rehabilitation.

Thanking you,

Dr Kailu Liang, Dr Xiaoling Gan, Prof Zhongliang Deng, Prof Zhou Xiang, Prof Fuguo Huang

\section{References}

1. Kailu L, Zhou X, Fuguo H (2010) Chronic perilunate dislocations treated with open reduction and internal fixation: results of medium-term follow-up. Int Orthop 34(8):1315-1320

2. Geissler WB, Freeland AE, Savoie FH, McIntyre LW, Whipple TL (1996) Intracarpal soft-tissue lesions associated with an intra-articular fracture of the distal end of the radius. J Bone Joint Surg Am 78 (3):357-365

3. Mayfield JK (1984) Patterns of injury to carpal ligaments: a spectrum. Clin Orthop Relat Res 187:36-42 